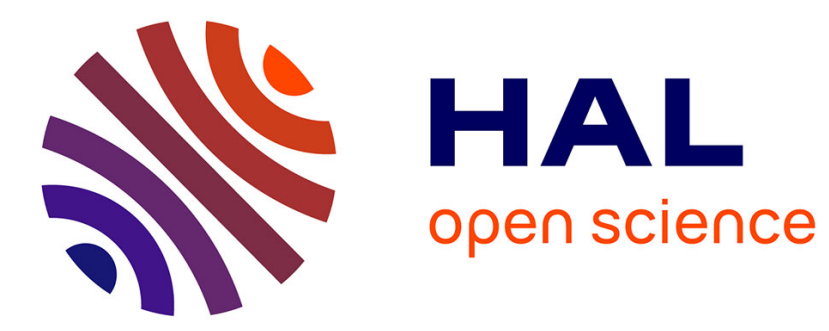

\title{
The growth ratio of synchronous rational relations is unique
}

Olivier Carton

\section{To cite this version:}

Olivier Carton. The growth ratio of synchronous rational relations is unique. Theoretical Computer Science, 2007, 376, pp.52-59. hal-00160891

\section{HAL Id: hal-00160891 https://hal.science/hal-00160891}

Submitted on 9 Jul 2007

HAL is a multi-disciplinary open access archive for the deposit and dissemination of scientific research documents, whether they are published or not. The documents may come from teaching and research institutions in France or abroad, or from public or private research centers.
L'archive ouverte pluridisciplinaire HAL, est destinée au dépôt et à la diffusion de documents scientifiques de niveau recherche, publiés ou non, émanant des établissements d'enseignement et de recherche français ou étrangers, des laboratoires publics ou privés. 


\title{
The growth ratio of synchronous
} rational relations is unique

\author{
Olivier Carton* \\ http://www.liafa.jussieu.fr/ carton
}

July 9,2007

\begin{abstract}
We introduce $\alpha$-synchronous relations for a rational number $\alpha$. We show that if a rational relation is both $\alpha$ - and $\alpha^{\prime}$-synchronous for two different numbers $\alpha$ and $\alpha^{\prime}$, then it is recognizable. We give a synchronization algorithm for $\alpha$-synchronous transducers. We also prove the closure under boolean operations and composition of $\alpha$-synchronous relations.
\end{abstract}

\section{Introduction}

We introduce $\alpha$-synchronous relations for a rational number $\alpha$. They extend the classical notion of synchronous relations, which are rational relations realized by letter to letter transducers. In these usual synchronous transducers, the ratio between the output length and the input length is always 1 whereas we allow it to be any fixed rational number $\alpha$. These relations have already been mentioned by Sakarovitch [8, p. 660].

The main result about these $\alpha$-synchronous relations is a Cobham-like theorem. We show that if a relation is both $\alpha$ - and $\alpha^{\prime}$-synchronous for two distinct rational number $\alpha$ and $\alpha^{\prime}$, then it is recognizable. This question was raised by Sakarovitch [8, p. 660]. Recall that Cobham's result states that if the base $k$ representation of a set of integers is regular for two multiplicatively independent bases $k$, it is ultimately periodic [2]. If $k$ and $k^{\prime}$ are multiplicatively independent, conversion from base $k$ to base $k^{\prime}$ is not rational. Otherwise, this conversion is a $\left(\log k^{\prime} / \log k\right)$-synchronous relation.

We also study the synchronization of transducers. We show that if a relation is realized by a transducer in which the ratio between the output length and the input length is $\alpha$ for any cycle, it can be also realized by another transducer in which the ratio between the output length and the

${ }^{*}$ LIAFA, Université Paris 7 \& CNRS, 2, pl. Jussieu - 75251 Paris Cedex - 05 
input length is $\alpha$ for any transition. This algorithm can be viewed as a normalization of $\alpha$-synchronous transducers.

The question of the synchronization of transducers goes back to the paper of Elgot and Mezei [4] about rational relations realized by finite automata, and to the result of Eilenberg and Schützenberger [3], which states that a length preserving rational relation of $A^{*} \times B^{*}$ is a rational subset of $(A \times B)^{*}$, or, equivalently, is realized by a synchronous automaton. The proof of Eilenberg is effective but is done on regular expressions and not directly on automata. In [5], Frougny and Sakarovitch give an algorithm for synchronization of relations with bounded length difference, the relations being between finite words or between one-sided infinite words. This constitutes another proof of Eilenberg and Schützenberger's result. Their algorithm operates directly on the transducer that realizes the relation. Our synchronization algorithm is an extension to $\alpha$-synchronous transducer of the algorithm given in [5].

One main ingredient of the synchronization algorithm is the state splitting transformation. The notion of state splitting, which appeared early in information theory, has been introduced to symbolic dynamics by Williams. Since then, it has been widely used, for example to solve certain coding problems $[7,1]$.

Finally, we show that the class of $\alpha$-synchronous relations is a boolean algebra as in the case of classical synchronous relations. This result is a crucial property in the theory of automatic structures [6].

The paper is organized as follows. Section 2 introduces the basic definitions of rational relations and transducers. The $\alpha$-synchronous transducers and relations are defined in Section 3. The synchronization algorithm is described in Section 4. Some closure properties of these relations are proved in section 5 and the main theorem is proved in Section 6 .

\section{Preliminaries}

In what follows, $A$ and $B$ denote finite alphabets. The free monoid $A^{*}$ is the set of finite words or sequences of letters from $A$. The empty word is denoted by $\varepsilon$. The length of a word $u \in A^{*}$ is denoted by $|u|$. In this paper, we study relations, that is, subsets of the product monoid $A^{*} \times B^{*}$. For a relation $R \subseteq A^{*} \times B^{*}$, we denote by $R^{-1}$ the relation $\{(v, u) \mid(u, v) \in R\}$.

A transducer (also known as a two-tape automaton) is a non-deterministic automaton whose transitions are labeled by pairs of words. A transducer over the monoid $A^{*} \times B^{*}$ is composed of a finite set $Q$ of states, a finite set $E \subset Q \times A^{*} \times B^{*} \times Q$ of transitions and two sets $I, F \subseteq Q$ of initial and final states. A transition $\tau=(s, u, v, t)$ from $s$ to $t$ is denoted by $s \stackrel{u \mid v}{\longrightarrow} t$.

A path in a transducer $\mathcal{T}$ is a sequence

$$
s_{0} \stackrel{u_{0} \mid v_{0}}{\longrightarrow} s_{1} \stackrel{u_{1} \mid v_{1}}{\longrightarrow} \cdots \stackrel{u_{n} \mid v_{n}}{\longrightarrow} s_{n}
$$


of consecutive transitions. The label of this path is the pair $(u, v)$ where its input label $u$ is the word $u_{1} u_{2} \cdots u_{n}$ and its output label $v$ is the word $v_{1} v_{2} \cdots v_{n}$. Such a path is denoted $s_{0} \stackrel{u \mid v}{\longrightarrow} s_{n}$. This path is accepting if $s_{0}$ is initial and $s_{n}$ is final. The set accepted by the transducer is the set of labels of its accepting paths, which is a relation $R \subseteq A^{*} \times B^{*}$. We say that the relation $R$ is realized by the transducer $\mathcal{T}$.

A subset of $A^{*} \times B^{*}$ is rational if it can be obtained from some finite subsets using union, concatenation and star iteration. It is a consequence of Kleene's theorem that a subset of $A^{*} \times B^{*}$ is a rational relation if and only if it is the relation realized by a transducer.

We now recall the definition of a class of very simple rational relations. A relation $R \subseteq A^{*} \times B^{*}$ is recognizable if there are two families $K_{1}, \ldots, K_{n}$ and $L_{1}, \ldots, L_{n}$ of rational subsets of $A^{*}$ and $B^{*}$ such that $R=\bigcup_{i=1}^{n} K_{i} \times L_{i}$.

\section{$3 \quad \alpha$-synchronous relations}

In this section, we first define the notion of $\alpha$-synchronous transducer and $\alpha$-synchronous relation. Through the section, a positive rational number $\alpha=p / q$ is fixed. We assume that the integers $p$ and $q$ are relatively prime.

A transducer $\mathcal{T}$ is $\alpha$-synchronous if for each transition $s \stackrel{u \mid v}{\longrightarrow} t$, the lengths of the input and output labels satisfy $|u|=q$ and $|v|=p$. If follows immediately that $\alpha$ is the ratio between the output length and input length of any path, that is, for any path $s \stackrel{u \mid v}{\longrightarrow} t$ in $\mathcal{T}$, the equality $|v| /|u|=\alpha$ holds.

Let \# be a padding symbol that does not belong to the alphabets $A$ and $B$. The padding of a pair $(u, v)$ of words over $A$ and $B$ is the pair $\left(u \#^{m}, v \#^{n}\right)$ where $m$ and $n$ are the least integers such that $\left|v \#^{n}\right| /\left|u \#^{m}\right|=$ $\alpha$. The integers $m$ and $n$ are actually given by $m=q r-|u|$ and $n=p r-|v|$ where the integer $r$ is defined by $r=\max (\lceil|u| / q\rceil,\lceil|v| / p\rceil)$. The padding of $(u, v)$ is denoted $(u, v)^{\#}$ without any reference to $\alpha$ although it depends on $\alpha$. For a relation $R \subseteq A^{*} \times B^{*}$, we denote by $R^{\#}$ the following relation

$$
R^{\#}=\left\{(u, v)^{\#} \mid(u, v) \in R\right\} .
$$

A relation $R \subseteq A^{*} \times B^{*}$ is $\alpha$-synchronous if the relation $R^{\#}$ can be realized by an $\alpha$-synchronous transducer over the alphabets $A \cup\{\#\}$ and $B \cup\{\#\}$.

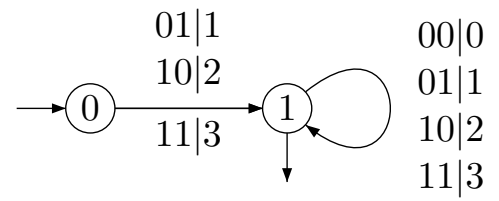

Figure 1: Conversion from base 2 to base 4 
Example 1. The transducer of Figure 1 performs conversion of the integers from base 2 to base 4 . Indeed, its accepts the pairs $(u, v)$ where $u$ is the base- 2 expansion of some integer $n$ (padded with a leading 0 to make it of even length) and $v$ is the base- 4 expansion of $n$. This transducer is $1 / 2$ synchronous.

\section{Synchronization}

A transducer has the $\alpha$-cycle property if for any cycle $s \stackrel{u \mid v}{\longrightarrow} s$, the equality $|v| /|u|=\alpha$ holds. Of course, an $\alpha$-synchronous transducer satisfies the $\alpha$ cycle property. The property of being $\alpha$-synchronous is a local property whereas the $\alpha$-cycle property is a global one. The following proposition states that these two properties are in fact equivalent.

Proposition 2. A relation realized by a transducer with the $\alpha$-cycle property is $\alpha$-synchronous.

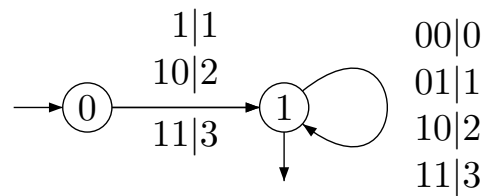

Figure 2: Conversion from base 2 to base 4 (cont.)

Example 3. In the transducer of Figure 1, a leading 0 has been added in the transition $0 \stackrel{01 \mid 1}{\longrightarrow} 1$ to make the transducer $1 / 2$-synchronous. If this 0 is removed, this yields the transducer of Figure 2, which is not $1 / 2$-synchronous but has the $1 / 2$-cycle property.

The rest of this section is devoted to the proof of the proposition. The proof is separated into several lemmas.

Lemma 4. Let $\alpha=p / q$ be a rational number. For any transducer $\mathcal{T}$ with the $\alpha$-cycle property, there is a constant $K$ such that the inequality $|q| v|-p| u|| \leq$ $K$ holds for any path $s \stackrel{u \mid v}{\longrightarrow} t$ in $\mathcal{T}$.

Proof. Let $K^{\prime}$ be defined by $K^{\prime}=\max \{|u|+|v| \mid s \stackrel{u \mid v}{\longrightarrow} t \in E\}$ where $E$ is the set of transitions of $\mathcal{T}$. Let now $K$ be defined

$$
K=K^{\prime}|Q| \max (p, q),
$$

where $|Q|$ is the number of states of $\mathcal{T}$. We claim that $K$ has the required property. 
Let $\gamma$ be a path $s \stackrel{u \mid v}{\longrightarrow} t$. If the length of $\gamma$ is less than $|Q|$, then $|q| v|-p| u|| \leq \max (p, q)(|u|+|v|) \leq K$. If the length of $\gamma$ is greater than $|Q|$, the path $\gamma$ can be factorized

$$
s \stackrel{u_{1} \mid v_{1}}{\longrightarrow} s^{\prime} \stackrel{u_{2} \mid v_{2}}{\longrightarrow} s^{\prime} \stackrel{u_{3} \mid v_{3}}{\longrightarrow} t
$$

where $u=u_{1} u_{2} u_{3}$ and $v=v_{1} v_{2} v_{3}$. One has $q|v|-p|u|=q\left|v_{1} v_{3}\right|-p\left|u_{1} u_{3}\right|$ since $q\left|v_{2}\right|-p\left|u_{2}\right|=0$. The result follows now from an easy induction on the length of the path.

We say that a transducer has the $\alpha$-balance property if for any two paths $s \stackrel{u \mid v}{\longrightarrow} t$ and $s \stackrel{u^{\prime} \mid v^{\prime}}{\longrightarrow} t$ with the same starting and ending states, the equality $q|v|-p|u|=q\left|v^{\prime}\right|-p\left|u^{\prime}\right|$ holds. Note that the $\alpha$-balance property is stronger than the $\alpha$-cycle property since we always assume an empty path from $s$ to $s$ labeled by the pair $(\varepsilon, \varepsilon)$. The following lemma states that they are in fact equivalent.

Lemma 5. Let $\alpha=p / q$ be a rational number. For any transducer $\mathcal{T}$ with the $\alpha$-cycle property, there is a transducer $\mathcal{T}^{\prime}$ with the $\alpha$-balance property and a single initial state that realizes the same relation as $\mathcal{T}$.

Proof. We may assume that $\mathcal{T}$ has a single initial state. If $\mathcal{T}$ has several initial states, a new state, which becomes the unique initial state is added to $\mathcal{T}$. Since no cycle is added by this operation, the $\alpha$-cycle property is not changed.

By the previous lemma, there is a constant $K$ such that the inequality $|q| v|-p| u|| \leq K$ holds for any path $s \stackrel{u \mid v}{\longrightarrow} t$ in $\mathcal{T}$. We construct a transducer $\mathcal{T}^{\prime}$ whose state set is $Q \times\{-K, \ldots,+K\}$ where $Q$ is the state set of $\mathcal{T}$. The initial state is $(i, 0)$ and the set of final states is $F \times\{-K, \ldots,+K\}$. The transitions are the transitions of the form $(s, \ell) \stackrel{u \mid v}{\longrightarrow}(t, m)$ where $s \stackrel{u \mid v}{\longrightarrow} t$ is transition of $\mathcal{T}$ and $m=\ell+q|v|-p|u|$.

For any path

$$
s_{0} \stackrel{u_{0} \mid v_{0}}{\longrightarrow} s_{1} \stackrel{u_{1} \mid v_{1}}{\longrightarrow} \cdots \stackrel{u_{n} \mid v_{n}}{\longrightarrow} s_{n}
$$

in $\mathcal{T}$, there is a path

$$
\left(s_{0}, m_{0}\right) \stackrel{u_{0} \mid v_{0}}{\longrightarrow}\left(s_{1}, m_{1}\right) \stackrel{u_{1} \mid v_{1}}{\longrightarrow} \cdots \stackrel{u_{n} \mid v_{n}}{\longrightarrow}\left(s_{n}, m_{n}\right)
$$

in $\mathcal{T}^{\prime}$ where $m_{0}=0$ and $m_{i}=q\left|v_{0} \cdots v_{i-1}\right|-p\left|u_{0} \cdots u_{i-1}\right|$ for $1 \leq i \leq n$. Conversely, any such path in $\mathcal{T}^{\prime}$ comes from a path in $\mathcal{T}$. This proves that $\mathcal{T}$ and $\mathcal{T}^{\prime}$ realize the same relation. Furthermore, the transducer $\mathcal{T}^{\prime}$ has the $\alpha$-balance property by construction.

Example 6. The transducer of Figure 2 has the $1 / 2$-cycle property but it does not have the $1 / 2$-balance property. If the construction given in the proof of the lemma is applied to this transducer, one gets the transducer of Figure 3 , which has the 1/2-balance property. 


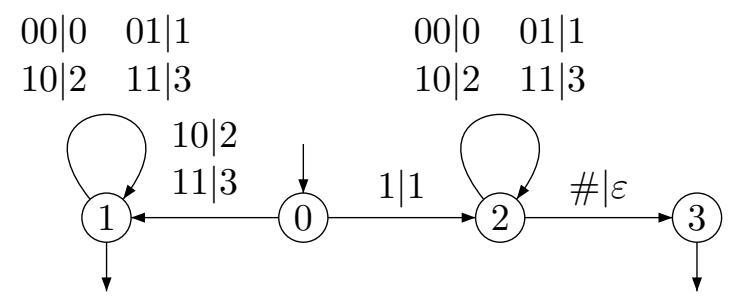

Figure 3: Conversion from base 2 to base 4 (cont.)

Let $\alpha=p / q$ be a rational number and let $\mathcal{T}$ be a transducer with the $\alpha$-balance property and a single initial state $i$. Define the function $b$ from $Q$ to $\mathbb{N}$ by $b(s)=q|v|-p|u|$ where $i \stackrel{u \mid v}{\longrightarrow} s$ is a path from $i$ to $s$. The $\alpha$-balance property ensures that the value $q|v|-p|u|$ does not depend on the path and the function $b$ is well-defined. The value $b(s)$ is called the balance of the state $s$. The function $b$ has the following properties. The balance of the initial state is 0 and for any path $s \stackrel{u \mid v}{\longrightarrow} t, q|v|-p|u|$ is equal to $b(t)-b(s)$.

If a transducer $\mathcal{T}$ has the property that the balance of any state is 0 , the label of any transition is a pair $(u, v)$ such that $q|v|-p|u|$ equals 0 . Since $p$ and $q$ are relatively prime, there is an integer $k$ such that $|u|=k q$ and $|v|=k p$. If $k$ equals 0 , the label of the transition is $(\varepsilon, \varepsilon)$. These transitions can be removed using standard techniques to remove $\varepsilon$-transitions. If $k \geq 2$, the words $u$ and $v$ can be factorized $u=u_{1} \cdots u_{k}$ where $\left|u_{i}\right|=q$ and $v=v_{1} \cdots v_{k}$ where $\left|v_{i}\right|=p$. The transition $s \stackrel{u \mid v}{\longrightarrow} t$ can be replaced by the $k$ transitions $s_{i-1} \stackrel{u_{i} \mid v_{i}}{\longrightarrow} s_{i}$ where $s_{0}=s, s_{k}=t$ and $s_{1}, \ldots, s_{k-1}$ are newly introduced states. Applying this transformation to each transition yields an $\alpha$-synchronous transducer that realizes the same relation.

Once a transducer with the $\alpha$-balance property has been obtained, it must be ensured that all final states have a 0-balance. Some final states may have a balance different from 0 since a pair $(u, v) \in R$ may not satisfy $|v| /|u|=\alpha$. Since any pair $(u, v)^{\#} \in R^{\#}$ satisfies this equality, the transducer must be slightly transformed. A new state $f$, which becomes the unique final state is added. Furthermore, for any former final $s$ state with balance $q a-b p$, a new transition $s \stackrel{\#^{m} \mid \#^{n}}{\longrightarrow} f$ is also added where $m=q r-b$, $n=p r-a$ and $r=\max (\lceil b / q\rceil,\lceil a / p\rceil)$.

Let $\alpha=p / q$ be a rational number. Since $p$ and $q$ are relatively prime, any integer $n$ is equal to $q a-p b$ for some integers $a$ and $b$. Furthermore, both integers $a$ and $b$ can be chosen positive since $n$ is also equal to $q(a+$ $k p)-p(b+k q)$ for any integer $k$. For any integer $n$, let us define $\|n\|$ as follows.

$$
\|n\|=\min \{a+b \mid n=q a-p b \text { with } a, b \geq 0\} .
$$

We call $\|n\|$ the weight of $n$. It is true that $\|n\|=0$ if and only if $n=0$ but the weight of $-n$ is not necessarily equal to the weight of $n$. Note that 
if $n=q a-p b$ and $\|n\|=a+b$, then $a<p$ or $b<q$ holds. Otherwise $n=q a^{\prime}-p b^{\prime}$ where $a^{\prime}=a-p$ and $b^{\prime}=b-q$ and $a^{\prime}+b^{\prime}<a+b$.

Example 7. If $\alpha=2 / 3$, the weight of some small integers is the following.

\begin{tabular}{c|cc|c|c|c|c|c|c|c|c|c|c|cc}
$n$ & $\cdots$ & -5 & -4 & -3 & -2 & -1 & 0 & 1 & 2 & 3 & 4 & 5 & 6 & $\cdots$ \\
\hline$\|n\|$ & & 5 & 2 & 4 & 1 & 3 & 0 & 2 & 4 & 1 & 3 & 5 & 2 &
\end{tabular}

For a transducer $\mathcal{T}$ with the $\alpha$-balance property, we denote by $\|\mathcal{T}\|$ the integer $\max _{s \in Q}\|b(s)\|$. Note that $\|\mathcal{T}\|$ equals 0 if and only the balance of any state is 0 .

The proof of the next lemma is based on two transformations of transducers called state splitting and letter shifting that we now introduce. We first define the operation of out-state splitting in a transducer $\mathcal{T}$. Let $s$ be a state of $\mathcal{T}$ and let $O=\left(O_{1}, \ldots, O_{n}\right)$ be a partition of all transitions starting in $s$. The operation of out-state splitting relative to the partition $O$ transforms $\mathcal{T}$ into the transducer $\mathcal{T}^{\prime}$ where $Q^{\prime}=(Q \backslash\{s\}) \cup\left\{s_{1}, \ldots, s_{n}\right\}$ is obtained from $Q$ by splitting state $s$ into $n$ new states $s_{1}, \ldots, s_{n}$.

- all transitions of $\mathcal{T}$ that are not incident to $s$ are left unchanged.

- each $s_{i}$ has a copy of the transitions ending in $s$.

- the transitions starting in $s$ are distributed among $s_{1}, \ldots, s_{n}$ according to the partition $O$.

Note that transitions from $s$ to $s$ are considered as both starting and ending in $s$. Note also that the balance of the new states $s_{1}, \ldots, s_{n}$ is the same as the balance of $s$. The operation of in-state splitting is obtained by reversing the roles played by transitions ending and starting in $s$.

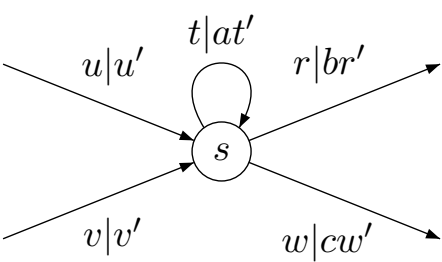

Before

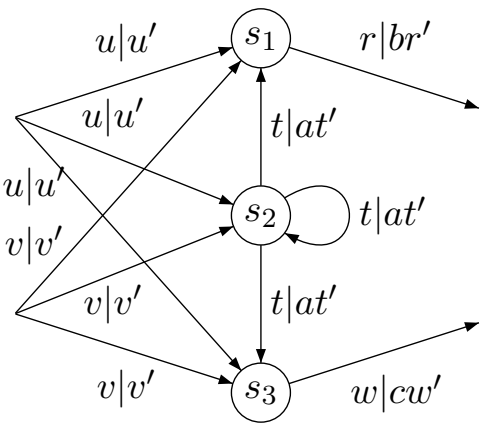

After

Figure 4: An out-state splitting

An out-state splitting is shown in Figure 4. There are three transitions $\tau_{1}, \tau_{2}$ and $\tau_{3}$, starting in the state $s$. The state $s$ is split according to the partition $O=\left(\left\{\tau_{1}\right\},\left\{\tau_{2}\right\},\left\{\tau_{3}\right\}\right)$ into three states $s_{1}, s_{2}$ and $s_{3}$.

We now describe the letter shifting operation. There are actually four variants whether input or output labels are considered and whether letters 


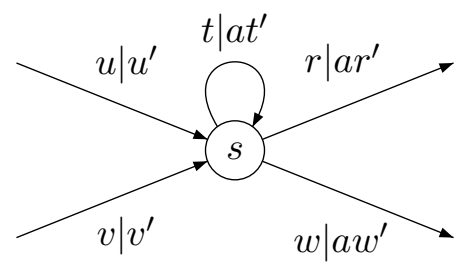

Before

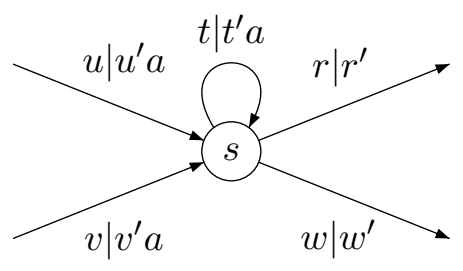

After

Figure 5: An output backwards shifting.

are shifted forwards or backwards. We describe the backwards shifting of the output letters. Let $s$ be state that is neither initial nor final and such that all outgoing transitions have a non-empty output label and that all these output labels start with the same letter $a$. If these conditions are not fulfilled, the operation cannot be performed. This letter $a$ is removed from the output labels of all outgoing transitions and it is added as the last letter of the output labels of all ingoing transitions. Note that transitions from $s$ to $s$ are considered as both starting and ending in $s$. The balance of the state $s$ is changed by the letter shifting. If its balance is $r$ before the operation, it becomes $r+q$ after an output backwards shifting and $r-p$ after an input backwards shifting. An output backwards shifting is shown in Figure 5.

Lemma 8. Let $\mathcal{A}$ be transducer with the $\alpha$-balance property such that the balance of any final state is 0 . If $\|\mathcal{T}\|>0$, there is a transducer $\mathcal{T}^{\prime}$ realizing the same relation and such that $\left\|\mathcal{T}^{\prime}\right\|<\|\mathcal{T}\|$.

Proof. It suffices to prove that the number of states $s$ such that $\|b(s)\|$ is maximal can be decreased. If this can be done, this number can be made equal to 0 and the value of $\|\mathcal{T}\|$ becomes strictly smaller.

In what follows, we call an $(\varepsilon, \varepsilon)$-transition a transition labeled by $(\varepsilon, \varepsilon)$ and an $(\varepsilon, \varepsilon)$-cycle a cycle made of $(\varepsilon, \varepsilon)$-transitions.

We claim that there is a state $s$ such that $\|b(s)\|$ is maximal and that there is no $(\varepsilon, \varepsilon)$-transition starting in $s$. If there is in $\mathcal{T}$ an $(\varepsilon, \varepsilon)$-cycle, all the states along this cycle can be merged into a single state and the cycle is removed. This operation is performed until no $(\varepsilon, \varepsilon)$-cycle remains. This process must stop since it decreases the number of states. We now suppose that there is no $(\varepsilon, \varepsilon)$-cycle. Let $s$ be state such that $\|b(s)\|$ is maximal. If there is no $(\varepsilon, \varepsilon)$-transition $s \stackrel{\varepsilon \mid \varepsilon}{\longrightarrow} t$, the state $t$ has the same balance as $s$ and state $t$ can be taken in place of $s$. Since there is no $(\varepsilon, \varepsilon)$-cycle, this process must end up with a state $s$ with the required properties.

Let $s$ be a state such that $\|b(s)\|$ is maximal and that there is no $(\varepsilon, \varepsilon)$ transition starting in $s$. Since $\|b(s)\|$ is not zero, the state $s$ is neither initial nor final. Let $a$ and $b$ be non-negative integers such that $b(s)=q a-p b$ and $\|b(s)\|=a+b$. Let $s \stackrel{u \mid v}{\longrightarrow} t$ be a transition starting in $s$. The balance 
of $t$ is $q(a+|v|)-p(b+|u|)$. Since $\|b(t)\| \leq\|b(s)\|$, one has $a+|v| \geq p$ and $b+|u| \geq q$. Otherwise, the weight of $b(t)$ would be $a+b+|u|+|v|$. Let $\ell$ and $m$ be defined by $\ell=\max (0, q-b)$ and $m=\max (0, p-a)$. Note that $(\ell, m)$ is different from $(0,0)$ since $a+b$ is the weight of $b(s)$. We have proved that for any transition $s \stackrel{u \mid v}{\longrightarrow} t$, both inequalities $|u| \geq \ell$ and $|v| \geq m$ hold.

Let $O=\left(O_{1}, \ldots, O_{n}\right)$ be a partition of the transitions starting in $s$ such that for any two transitions $s \stackrel{u \mid v}{\longrightarrow} t$ and $s \stackrel{u^{\prime} \mid v^{\prime}}{\longrightarrow} t^{\prime}$ in the part $O_{i}$, the first $\ell$ letters of $u$ and $u^{\prime}$ coincide and the first $m$ letters of $v$ and $v^{\prime}$ also coincide. The state $s$ is split into $n$ states $s_{1}, \ldots, s_{n}$ using an out-state splitting relative to the partition $O$. Each state $s_{i}$ has the same balance $a+b$ as $s$. Furthermore, all the transitions starting in $s$ have their input label with a common prefix of length $\ell$ and their input label with a common prefix of length $m$. For each $s_{i}$, $\ell$ letter from the input labels and $m$ letters from the output labels an be backwards shifted. The balance of $s_{i}$ becomes $q(a+m)-p(b+\ell)$. Since $a+m \geq p$ and $b+\ell \geq q$, let the non-negative integers $a^{\prime}$ and $b^{\prime}$ be defined by $a+m=a^{\prime}+p$ and $b+\ell=b^{\prime}+q$. Then the balance of $s_{i}$ is $q a^{\prime}-p b^{\prime}$. It is straightforward to verify that $a^{\prime}+b^{\prime}<a+b$ and that $\left\|b\left(s_{i}\right)\right\|$ has decreased.

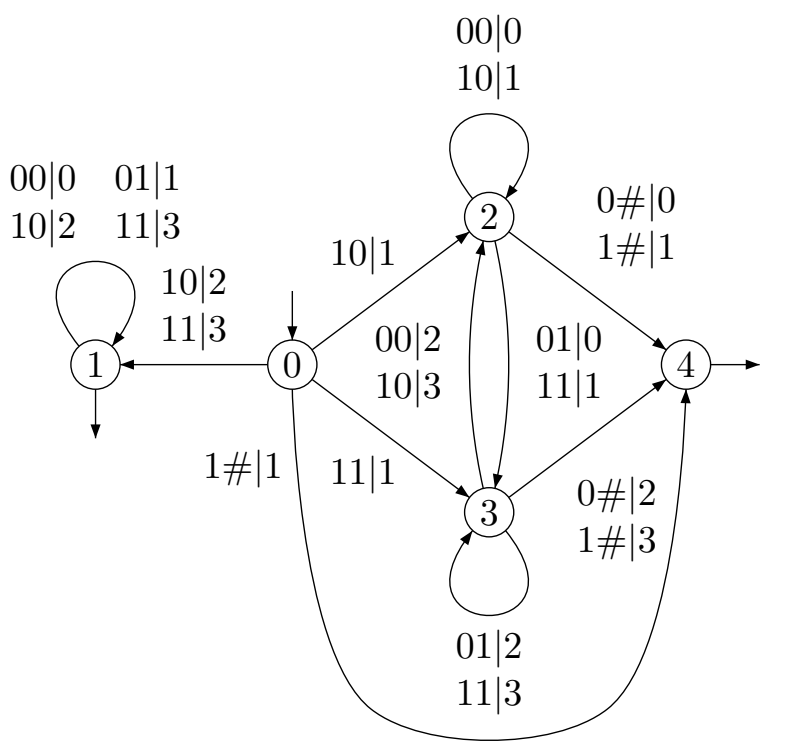

Figure 6: Conversion from base 2 to base 4 (cont.)

Example 9. If the proof of the proposition is applied to the transducer of Figure 3, one gets the transducer of Figure 6 . State 2 has been split into states 2,3 and 4 and there has been one backwards input shifting. 


\section{Closure properties}

The class of $\alpha$-synchronous relations is closed under several operations. Here, we prove the closure properties that are needed in the proof of the main theorem. We consider only the boolean operations and composition.

If $R$ and $R^{\prime}$ are two $\alpha$-synchronous relations (for the same $\alpha$ ), it is clear that the relation $R \cup R^{\prime}$ is also $\alpha$-synchronous. For a relation $R$, we denote by $\check{R}$ the complement relation $\{(u, v) \mid(u, v) \notin R\}$.

Proposition 10. If the relation $R$ is $\alpha$-synchronous, the relation $\check{R}$ is also $\alpha$-synchronous.

Proof. Let $\mathcal{T}$ be an $\alpha$-synchronous transducer realizing the relation $R^{\#}$. Since each transition of $\mathcal{T}$ is labeled by a pair $(u, v)$ of words such that $|u|=q$ and $|v|=p$, the transducer $\mathcal{T}$ can be viewed as an automaton over the alphabet $C=(A \cup\{\#\})^{q} \times(B \cup\{\#\})^{p}$. This automaton accepts a rational set of words $L$ over $C$. Let $\mathcal{T}^{\prime}$ be an automaton over $C$ accepting the complement of $L$. This automaton can be modified to accept only words of the form $A^{*} \#^{*} \times B^{*} \#^{*}$. This modified automaton over $C$ is actually an $\alpha$-synchronous transducer realizing $\check{R}^{\#}$.

For two relations $R \subseteq A^{*} \times B^{*}$ and $R^{\prime} \subseteq B^{*} \times C^{*}$, we denote by $R R^{\prime}$ the relation obtained by composition of $R$ and $R^{\prime}$, that is, the relation $\left\{(u, w) \mid \exists v(u, v) \in R\right.$ and $\left.(v, w) \in R^{\prime}\right\}$.

Proposition 11. If the relations $R \subseteq A^{*} \times B^{*}$ and $R^{\prime} \subseteq B^{*} \times C^{*}$ are respectively $\alpha$-and $\alpha^{\prime}$-synchronous, the relation $R R^{\prime}$ is $\alpha \alpha^{\prime}$-synchronous.

Proof. Let $\mathcal{T}$ be an $\alpha$-synchronous transducer realizing $R^{\#}$ and $\mathcal{T}^{\prime}$ be an $\alpha^{\prime}$ synchronous transducer realizing $R^{\prime \#}$. The following operation is performed on $\mathcal{T}$ and $\mathcal{T}^{\prime}$ to get the transducers $\mathcal{S}$ and $\mathcal{S}^{\prime}$. Each transition $s \stackrel{u \mid v}{\longrightarrow} t$ where $u=u_{1} \cdots u_{q}$ and $v=v_{1} \cdots v_{p}$ is replaced by the following path of length $q+p$

$$
s_{0} \stackrel{u_{1} \mid \varepsilon}{\longrightarrow} s_{1} \stackrel{u_{2} \mid \varepsilon}{\longrightarrow} \cdots \stackrel{u_{q} \mid \varepsilon}{\longrightarrow} s_{q} \stackrel{\varepsilon \mid v_{1}}{\longrightarrow} s_{q+1} \stackrel{\varepsilon \mid v_{2}}{\longrightarrow} \cdots \stackrel{\varepsilon \mid v_{p}}{\longrightarrow} s_{q+p}
$$

where $s_{0}=s, s_{q+p}=t$ and $s_{1}, \ldots, s_{q+p-1}$ are the newly introduced states. The transducers $\mathcal{S}$ and $\mathcal{S}^{\prime}$ still realize the relations $R^{\#}$ and $R^{\prime \#}$. They are no longer $\alpha$ - and $\alpha^{\prime}$-synchronous but they have the $\alpha$ - and $\alpha^{\prime}$-cycle properties. A transducer $\mathcal{R}$ realizing $\left(R R^{\prime}\right)^{\#}$ is defined as follows. Its set of states is $Q \times Q^{\prime}$ where $Q$ and $Q^{\prime}$ are the sets of states of $\mathcal{S}$ and $\mathcal{S}^{\prime}$. Its initial and final states are $I \times I^{\prime}$ and $F \times F^{\prime}$ where $I, F, I^{\prime}$ and $F^{\prime}$ are the initial and final set of states of $\mathcal{S}$ and $\mathcal{S}^{\prime}$. If $E$ and $E^{\prime}$ are the sets of transitions of $\mathcal{S}$ and $\mathcal{S}^{\prime}$, the transitions of $\mathcal{R}$ are defined as follows.

$$
\left\{\left(s, s^{\prime}\right) \stackrel{u \mid w}{\longrightarrow}\left(t, t^{\prime}\right) \mid \exists v \in B \cup\{\#, \varepsilon\} \quad s \stackrel{u \mid v}{\longrightarrow} t \in E \text { and } s^{\prime} \stackrel{v \mid w}{\longrightarrow} t^{\prime} \in E^{\prime}\right\}
$$


It is straightforward to verify that $\mathcal{R}$ realizes the relation $\left(R R^{\prime}\right)^{\#}$ and that it has the $\alpha \alpha^{\prime}$-cycle property. By Proposition 2, the relation $R R^{\prime}$ is $\alpha \alpha^{\prime}$ synchronous.

\section{Main theorem}

It is clear that a recognizable relation is $\alpha$-synchronous for any $\alpha>0$. The following theorem gives a converse.

Theorem 12. If a rational relation $R \subseteq A^{*} \times B^{*}$ is $\alpha$-and $\alpha^{\prime}$-synchronous for $\alpha \neq \alpha^{\prime}$, then $R$ is recognizable.

The proof of the theorem is based on the following lemma.

Lemma 13. Let $\sim$ be an equivalence relation on $A^{*}$ that is $\alpha$-synchronous for $\alpha \neq 1$. The relation $\sim$ has finite index (it has finitely many classes).

Proof. We claim that there is a constant $K$ such that if $w \in A^{*}$ satisfies $|w|>K$, there is $w^{\prime} \in A^{*}$ such that $w \sim w^{\prime}$ and $\left|w^{\prime}\right|<|w|$. This proves that the relation $\sim$ has finite index since the number of classes is bounded by the number of words of length smaller than $K$.

Since the relation $\sim^{-1}$ is equal to the relation $\sim$, we may assume that $\alpha=p / q>1$. Let $\mathcal{T}$ be an $\alpha$-synchronous transducer realizing the relation $\sim$. For each state $s$ of $\mathcal{T}$, we define $c(s)$ by

$$
c(s)=\min \left\{|u| \mid s \stackrel{u \mid v}{\longrightarrow} f \text { is a path where } v \in \#^{*} \text { and } f \text { is final }\right\} .
$$

By convention, we set $c(s)=\infty$ if no such word $u$ exists. Let $C$ and $K$ be defined by $C=\max _{c(s) \neq \infty} c(s)$ and $K=\alpha(p+C) /(1-\alpha)$.

Let $w$ be a word such that $w>K$. Since $w \sim w$ holds, there is an accepting path labeled by $(w, w)^{\#}$ in $\mathcal{T}$. This path can be decomposed

$$
i \stackrel{u \mid v}{\longrightarrow} s \stackrel{u^{\prime} \mid v^{\prime}}{\longrightarrow} f
$$

where $\left(u u^{\prime}, v v^{\prime}\right)=(w, w)^{\#}, v=w \#^{n}$ with $n<p$ and $v^{\prime} \in \#^{*}$. Since $|v| \leq|w|+p$ and $|v| /|u|=\alpha$. One has $|u|<p+|w| / \alpha$. By definition of $C$, there is a path $s \stackrel{u^{\prime \prime} \mid v^{\prime \prime}}{\longrightarrow} f^{\prime}$ such that $\left|u^{\prime \prime}\right| \leq C, v^{\prime \prime} \in \#^{*}$ and $f^{\prime}$ is a final state. Then there is an accepting path labeled by $\left(u u^{\prime \prime}, u v^{\prime \prime}\right)$ in $\mathcal{T}$. Furthermore, the word $u u^{\prime \prime}$ satisfies $\left|u v^{\prime \prime}\right| \leq|u|+C<p+C+|w| / \alpha \leq|w|$. There is a word $w^{\prime}$ such that $\left(u u^{\prime \prime}, v v^{\prime \prime}\right)=\left(w^{\prime}, w\right)^{\#}$ and $\left|w^{\prime}\right|<|w|$. We have found a word $w^{\prime}$ such that $w \sim w^{\prime}$ and $\left|w^{\prime}\right|<|w|$.

We come now to the proof of the main theorem. 
Proof. Let $R$ be a relation that is $\alpha$ - and $\alpha^{\prime}$-synchronous for $\alpha \neq \alpha^{\prime}$. For a word $u$ in $A^{*}$, we denote by $R(u)$ the set $\{v \mid(u, v) \in R\}$. We define the equivalence relation $\sim$ on $A^{*}$ as follows. For any word $u$ and $u^{\prime}$, the relation $u \sim u^{\prime}$ holds if and only if $R(u)=R\left(u^{\prime}\right)$. We claim that the relation $\sim$ is $\alpha / \alpha^{\prime}$-synchronous.

By Proposition 10, it suffices to prove that the relation $\nsim$ is $\alpha / \alpha^{\prime}$ synchronous. By definition of $\sim$, one has the following equivalence

$$
u \nsim u^{\prime} \Longleftrightarrow \exists v\left\{\begin{array}{c}
(u, v) \in R \wedge\left(u^{\prime}, v\right) \notin R \\
\vee \\
(u, v) \notin R \wedge\left(u^{\prime}, v\right) \in R
\end{array},\right.
$$

which shows that the relation $\not$ is equal to $R \check{R}^{-1} \cup \check{R} R^{-1}$ where $\check{R}=\{(u, v) \mid$ $(u, v) \notin R\}$. Since $R$ is $\alpha$-synchronous, the relation $\check{R}$ is also $\alpha$-synchronous by Proposition 10. Since $R$ is $\alpha^{\prime}$-synchronous, both relations $\check{R}^{-1}$ and $R^{-1}$ are also $1 / \alpha^{\prime}$-synchronous by Proposition 10. By Proposition 11, both relations $R \check{R}^{-1}$ and $\check{R} R^{-1}$ are $\alpha / \alpha^{\prime}$-synchronous.

By the previous lemma, the relation $\sim$ has finite index. This proves that $R$ is recognizable.

\section{References}

[1] R. L. Adler, D. Coppersmith, and M. Hassner. Algorithms for sliding block codes. IEEE Trans. Inform. Theory, IT-29:5-22, 1983.

[2] A. Cobham. On the base-dependance of sets of numbers recognizable by finite automata. Math. Systems Theor., 3:186-192, 1969.

[3] S. Eilenberg. Automata, Languages and Machines, volume A. Academic Press, New York, 1972.

[4] C. C. Elgot and J. E. Mezei. On relations defined by generalized finite automata. IBM Journal Res. and Dev., 9:47-68, 1965.

[5] Ch. Frougny and J. Sakarovitch. Synchronized relations of finite words. Theoret. Comput. Sci., 108:45-82, 1993.

[6] B. Khoussainov and S. Rubin. Automatic structures: Overview and future directions. Journal of Automata, Languages and Combinatorics, $8(2): 287-301,2003$.

[7] B. Marcus. Factors and extensions of full shifts. Monats. Math, 88:239247, 1979.

[8] J. Sakarovitch. Éléments de théorie des automates. Vuibert, 2003. 